\title{
Custo-efetividade dos análogos de prostaglandinas no Brasil
}

\section{Cost-effectiveness of prostaglandin analogues in Brazil}

Ricardo Augusto Paletta Guedes', Vanessa Maria Paletta Guedes², Alfredo Chaoubah ${ }^{3}$

\begin{abstract}
Resumo
Objetivo: Avaliar a relação custo-efetividade dos análogos das prostaglandinas para o tratamento do glaucoma e da hipertensão ocular no estado de Minas Gerais, Brasil. Métodos: Este estudo transversal avaliou o custo (preço máximo ao consumidor) das diferentes apresentações dos análogos de prostaglandinas (bimatoprosta, latanoprosta, travoprosta) em relação à sua efetividade na redução da pressão intra-ocular. Para cada uma das medicações, calculou-se o custo mensal, anual e em 5 anos para se obter uma redução percentual de $1 \%$ e $20 \%$ na pressão intra-ocular (PIO), assim como o custo mensal, anual e em 5 anos para se obter uma redução de $1 \mathrm{mmHg}$ e $8 \mathrm{mmHg}$ a partir da PIO inicial.Resultados: O custo mensal para se obter uma redução de $1 \%$ e $20 \%$ da PIO foi, respectivamente, de $\mathrm{R} \$ 1,35$ e $\mathrm{R} \$ 27,00$ para a bimatoprosta $5 \mathrm{ml}$, $\mathrm{R} \$ 1,50$ e $\mathrm{R} \$ 30,00$ para a bimatoprosta $3 \mathrm{ml}, \mathrm{R} \$ 1,53$ e $\mathrm{R} \$ 30,60$ para a travoprosta e $\mathrm{R} \$ 2,22$ e $\mathrm{R} \$ 44,40$ para a latanoprosta. O custo mensal máximo para uma redução de 1 e $8 \mathrm{mmHg}$ da PIO foi, respectivamente, de $\mathrm{R} \$ 6,01$ e $\mathrm{R} \$ 48,08$ para a bimatoprosta $5 \mathrm{ml}$, de $\mathrm{R} \$ 6,67$ e $\mathrm{R} \$ 53,36$ para a travoprosta, de $\mathrm{R} \$ 6,70$ e $\mathrm{R} \$ 53,60$ para a bimatoprosta $3 \mathrm{ml}$ e de $\mathrm{R} \$$ 9,83 e $\mathrm{R} \$ 78,64$ para a latanoprosta.Conclusão: A medicação mais custoefetividade foi a bimatoprosta, na apresentação de $5 \mathrm{ml}$. Aquela que se mostrou com a mais baixa relação custo-efetividade foi a latanoprosta. A travoprosta e a bimatoprosta na apresentação de $3 \mathrm{ml}$ apresentaram resultados semelhantes, ficando em posição intermediária entre as demais.
\end{abstract}

Descritores: Glaucoma/quimioterapia; Pressão intra-ocular/quimioterapia; Prostaglandinas sintéticas/economia; Custos de medicamentos; Avaliação de custoefetividade; Custos e análise de custos; Brasil

\footnotetext{
'Médico Oftalmologista do Centro Oftalmológico Paletta Guedes e Pesquisador da Universidade Federal de Juiz de Fora - UFJF - Juiz de Fora (MG), Brasil;

${ }^{2}$ Médica Oftalmologista do Centro Oftalmológico Paletta Guedes e Pesquisadora da Universidade Federal de Juiz de Fora - UFJF - Juiz de Fora (MG), Brasil;

'Professor do Departamento de Estatística de Pesquisador da Universidade Federal de Juiz de Fora - UFJF - Juiz de Fora (MG), Brasil.
} 


\section{INTRODUÇÃO}

$\mathbf{0}$ custo crescente da atenção à saúde tem se tornado um problema preocupante de saúde pública. Na oftalmologia, o glaucoma tem um impacto financeiro significativo para o sistema de saúde, pois envolve uso crônico de medicamentos, procedimentos cirúrgicos, consultas e exames complementares freqüentes. Isto, sem levar em conta os custos indiretos, os quais podem incluir: o gasto com o cuidador do deficiente visual e com a reabilitação, a incapacidade para o trabalho, etc ${ }^{(1)}$.

Os medicamentos constituem uma importante proporção dos custos diretos do glaucoma. Segundo Rylander e Vold, o custo anual com medicamentos nos EUA pode variar de 150,81 dólares até 873,98 dólares, se o paciente mantiver o uso de somente uma medicação.

Os principais tipos de estudo de custos econômicos em saúde incluem: análises de custo-minimização, de custo-efetividade, de custo-utilidade e de custo-benefício ${ }^{(2)}$. Análises de custo-efetividade e custo-benefício são estudos econômicos valiosos em saúde. A análise de custo-benefício avalia o custo de um produto ou de um serviço em relação a outros ${ }^{(3-4)}$. Por outro lado, a análise de custo-efetividade permite a comparação dos custos de um tratamento (em unidades monetárias) com os seus resultados ${ }^{(5)}$. Por isto, ela permite uma avaliação tanto em nível individual quanto em nível coletivo ${ }^{(5)}$.

O objetivo do presente estudo é avaliar e comparar a relação custo-efetividade do uso dos três análogos de prostaglandinas disponíveis no mercado brasileiro (especificamente em Minas Gerais) para tratamento do glaucoma e da hipertensão ocular.

\section{Métodos}

Este estudo transversal de farmacoeconomia avaliou o preço máximo ao consumidor, no mês de abril de 2008, (alíquota do Imposto sobre Circulação de Mercadorias e Serviços, ICMS, de 18\% para estado de Minas Gerais) das diferentes apresentações farmacológicas dos análogos de prostaglandinas disponíveis no mercado em relação a sua eficácia descrita na literatura.

As medicações estudadas foram: bimatoprosta 0,03\% (Lumigan ${ }^{\oplus}$,Allergan, Inc. Irvine, CA, EUA, frasco de $3 \mathrm{ml}$ ), bimatoprosta $0,03 \%$ (Lumigan ${ }^{\varpi}$, Allergan, Inc. Irvine, CA, EUA, frasco de $5 \mathrm{ml}$ ), latanoprosta $0,005 \%$ (Xalatan ${ }^{\varpi}$,Pfizer, Inc., New York, NY, EUA, frasco de $2,5 \mathrm{ml}$ ) e travoprosta $0,004 \%$ (Travatan ${ }^{\circledR}$, Alcon Laboratories, Inc. Ft. Worth, TX, EUA, frasco de 2,5 ml).

O custo diário, mensal, anual e em 5 anos do uso de cada medicação foi obtido por meio da divisão do custo (preço máximo ao consumidor, obtido do Guia da
Farmácia, de abril de 2008) pela duração em dias de cada colírio. A duração foi calculada pela média de gotas em cada frasco, dividida pelo número de gotas necessárias diariamente para o tratamento (1 gota para cada olho tratado por dia).

A média do número de gotas de cada frasco foi calculada a partir do cálculo da média aritmética do número de gotas de cada frasco obtida de quatro estudos prévios realizados no Brasil0 ${ }^{(6.9)}$. O cálculo do número médio de gotas da apresentação de $5 \mathrm{ml}$ da bimatoprosta $0,03 \%$ foi feito a partir do número médio de gotas na apresentação de $3 \mathrm{ml}$ por meio de proporcionalidade, pois o mesmo não tinha sido avaliado nos estudos citados anteriormente.

A eficácia dos medicamentos estudados foi obtida na literatura em duas formas diferentes: porcentagem de redução da pressão intra-ocular (PIO) e magnitude (em $\mathrm{mmHg}$ ) de redução da PIO proposta pelo próprio fabricante de cada medicação.

A porcentagem média de redução tensional de cada medicação foi extraída de uma meta-análise realizada por Denis et al. ${ }^{(10)}$, em que estes autores calcularam uma eficácia média dos três análogos de prostaglandinas disponíveis. Os autores do presente estudo decidiram utilizar esta mesma média, visto o rigor do cálculo realizado por aqueles autores.

A faixa de redução em mmHg da PIO foi obtida do estudo de Frenkel et al. Neste estudo, os autores extraíram da própria bula do fabricante nos EUA o nível de redução pressórica obtida para cada medicação ${ }^{(10)}$.

Custo-efetividade foi definido na análise como o custo mensal, anual e em 5 anos para reduzir a PIO em $1 \%$ e $20 \%$, assim como o custo mensal, anual e em 5 anos para reduzir a $\mathrm{PIO}$ em $1 \mathrm{mmHg}$ e $8 \mathrm{mmHg}$. O valor de $20 \%$ foi escolhido baseando-se no fato de que uma redução de $20 \%$ seria capaz de prevenir a progressão do hipertenso ocular e do glaucomatoso na maioria dos pacientes ${ }^{(12)}$. O valor de $8 \mathrm{mmHg}$ foi escolhido por ser o valor máximo alcançado pelos 3 medicamentos na redução da PIO ${ }^{(11)}$.

\section{Resultados}

O número médio de gotas de cada frasco pode ser visto na Tabela 1.

O preço máximo ao consumidor para uma alíquota de ICMS de $18 \%$ no mês de abril de 2008 foi $\mathrm{R} \$ 86,22$ para a bimatoprosta de $3 \mathrm{ml}, \mathrm{R} \$ 129,33$ para a bimatoprosta de $5 \mathrm{ml}, \mathrm{R} \$ 105,93$ para a latanoprosta e $\mathrm{R} \$ 79,75$ para a travoprosta. A Tabela 2 indica o preço máximo ao consumidor dos quatro medicamentos estudados, bem como a duração em dias do tratamento e o custo mensal, anual e em 5 anos para cada um dos colírios. A eficácia de cada medicamento em porcenta- 
Tabela 1

Número médio de gotas de cada frasco do medicamento estudado

\begin{tabular}{|c|c|c|c|c|c|c|}
\hline \multicolumn{7}{|c|}{ Estudos } \\
\hline Medicamentos & $\begin{array}{c}1 \\
\text { (Stillitano IG, } \\
\left.\text { et al. }^{6}\right)\end{array}$ & $\begin{array}{c}2 \\
\text { (Stillitano IG, } \\
\text { et al. }^{7} \text { ) }\end{array}$ & $\begin{array}{c}3 \\
(\text { Galvão-Neto } P \text {, } \\
{\text { et } \text { al }^{8} \text { ) }}^{\text {(G) }}\end{array}$ & $\begin{array}{c}4 \\
\text { (Galvão-Neto P, } \\
\left.\text { et al. }{ }^{\text {') }}\right)\end{array}$ & Média & DP \\
\hline Latanoprosta & 110,80 & 102,13 & 111,50 & 106,67 & 107,78 & 4,32 \\
\hline Travoprosta & 102,62 & 100,80 & 103,17 & 103,66 & 102,56 & 1,25 \\
\hline Bimatoprosta 3ml & 109,12 & 101,80 & 116,17 & 114,00 & 110,27 & 6,37 \\
\hline Bimatoprosta $5 \mathrm{ml}^{*}$ & 181,87 & 169,67 & 193,62 & 190,00 & 184,43 & 12,91 \\
\hline
\end{tabular}

*Cálculo da bimatoprosta de $5 \mathrm{ml}$ foi feito utilizando-se de proporcionalidade a partir do número de gotas no frasco de 3 ml; DP: DesvioPadrão

Tabela 2

Preço máximo ao consumidor, número de gotas em cada frasco, duração de cada frasco e custo mensal, anual e em 5 anos para cada medicação em estudo (Minas Gerais, abril de 2008)

\begin{tabular}{|c|c|c|c|c|c|c|}
\hline Medicamentos & $\begin{array}{c}\text { PMC } * \\
\text { (valor em reais) }\end{array}$ & $\begin{array}{l}N^{\circ} \text { de gotas } \\
(\mathrm{ml})\end{array}$ & $\begin{array}{r}\text { Duração } \\
\text { (dias)** }\end{array}$ & $\begin{array}{l}\text { Custo } \\
\text { mensal }\end{array}$ & $\begin{array}{l}\text { Custo } \\
\text { anual }\end{array}$ & $\begin{array}{c}\text { Custo } \\
\text { em } 5 \text { anos }\end{array}$ \\
\hline Latanoprosta $2,5 \mathrm{ml}$ & 105,93 & 107,78 & 53,89 & 58,97 & 707,64 & 3538,21 \\
\hline Travoprosta 2,5 ml & 79,75 & 102,56 & 51,28 & 46,66 & 559,87 & 2799,34 \\
\hline Bimatoprosta 3ml & 86,22 & 110,27 & 55,14 & 46,91 & 562,97 & 2814,84 \\
\hline Bimatoprosta $5 \mathrm{ml}$ & 129,33 & 184,43 & 92,22 & 42,07 & 504,89 & 2524,47 \\
\hline
\end{tabular}

* PMC: Preço Máximo ao Consumidor no mês de abril de 2008 com alíquota de 18\% de ICMS (estado de Minas Gerais);

** Duração de cada frasco, admitindo-se o uso de 2 gotas por dia, sem desperdício e com fidelidade de $100 \%$

gem e valores absolutos $(\mathrm{mmHg})$ de redução da $\mathrm{PIO}$ são mostradas na Tabela 3 .

A análise de custo-efetividade de cada medicamento pode ser vista nas Tabelas 4 e 5 e nos Gráficos 1 e 2. O custo mensal para se obter uma redução de $1 \%$ na PIO foi de R \$ 2,22 para a latanoprosta, $\mathrm{R} \$ 1,53$ para a travoprosta, $\mathrm{R} \$ 1,50$ para a bimatoprosta de $3 \mathrm{ml}$ e $\mathrm{R} \$$ 1,35 para a bimatoprosta de $5 \mathrm{ml}$. Para uma redução semelhante da PIO, o custo em 5 anos seria de $\mathrm{R} \$ 133,12$ para a latanoprosta, $\mathrm{R} \$ 91,93$ para a travoprosta, $\mathrm{R} \$$ 90,07 para a bimatoprosta de $3 \mathrm{ml}$ e $\mathrm{R} \$ 80,78$ para a bimatoprosta de $5 \mathrm{ml}$. Segundo este critério, a droga mais custo-efetiva seria a bimatoprosta de $5 \mathrm{ml}$, seguida pela bimatoprosta de $3 \mathrm{ml}$, pela travoprosta e pela latanoprosta.

O custo para uma redução de 1 e $8 \mathrm{mmHg}$ da PIO sofreu uma variação no cálculo de acordo com a faixa de redução pressórica em mmHg para cada medicação (bimatoprosta: 7 a $8 \mathrm{mmHg}$; latanoprosta: 6 a $8 \mathrm{mmHg}$ e travoprosta: 7 a $8 \mathrm{mmHg}$ ). Os valores (em reais) encontrados são resultados da divisão do custo (mensal, anual e em 5 anos) pelos valores mínimo e máximo da faixa de redução pressórica (em $\mathrm{mmHg}$ ). O custo mensal para reduzir $1 \mathrm{mmHg}$ variou de $\mathrm{R} \$ 7,37$ a $\mathrm{R} \$ 9,83$ para a latanoprosta, de $\mathrm{R} \$ 5,83$ a $\mathrm{R} \$ 6,67$ para a travoprosta, de
Tabela 3

Eficácia dos medicamentos estudados obtida na literatura

\begin{tabular}{lccc}
\hline \multirow{2}{*}{ Medicamentos } & $\begin{array}{c}\text { \% de redução } \\
\text { da PIO* }\end{array}$ & \multicolumn{2}{c}{$\begin{array}{c}\text { Redução da } \\
\text { PIO (mmHg)** }\end{array}$} \\
\cline { 3 - 4 } & & Mínimo & Máximo \\
\hline Latanoprosta & $26,58 \%$ & 6 & 8 \\
Travoprosta & $30,45 \%$ & 7 & 8 \\
Bimatoprosta de $3 \mathrm{ml}$ & $31,25 \%$ & 7 & 8 \\
Bimatoprosta de $5 \mathrm{ml}$ & $31,25 \%$ & 7 & 8 \\
\hline
\end{tabular}

* Porcentagem média de redução da PIO, segundo Denis et al. ${ }^{(10)}$; ** Limites de redução da PIO (mmHg), segundo Frenkel et al. ${ }^{(11)}$; $\mathrm{PIO}=$ Pressão intra-ocular

$\mathrm{R} \$ 5,86$ a $\mathrm{R} \$ 6,70$ para a bimatoprosta de $3 \mathrm{ml}$ e de $\mathrm{R} \$$ 5,26 a $\mathrm{R} \$ 6,01$ para a bimatoprosta de $5 \mathrm{ml}$. Se extrapolarmos estes valores a uma projeção de 5 anos, teremos os seguintes limites: de $\mathrm{R} \$ 442,28$ a $\mathrm{R} \$ 589,70$ para a latanoprosta, de $\mathrm{R} \$ 349,92$ a $\mathrm{R} \$ 399,91$ para a travoprosta, de $\mathrm{R} \$ 351,85$ a $\mathrm{R} \$ 402,12$ para a bimatoprosta de $3 \mathrm{ml}$ e de $\mathrm{R} \$ 315,56$ a $\mathrm{R} \$ 360,64$ para a bimatoprosta de $5 \mathrm{ml}$. De acordo com este critério de avaliação e comparação, a medicação mais custo-efeti- 

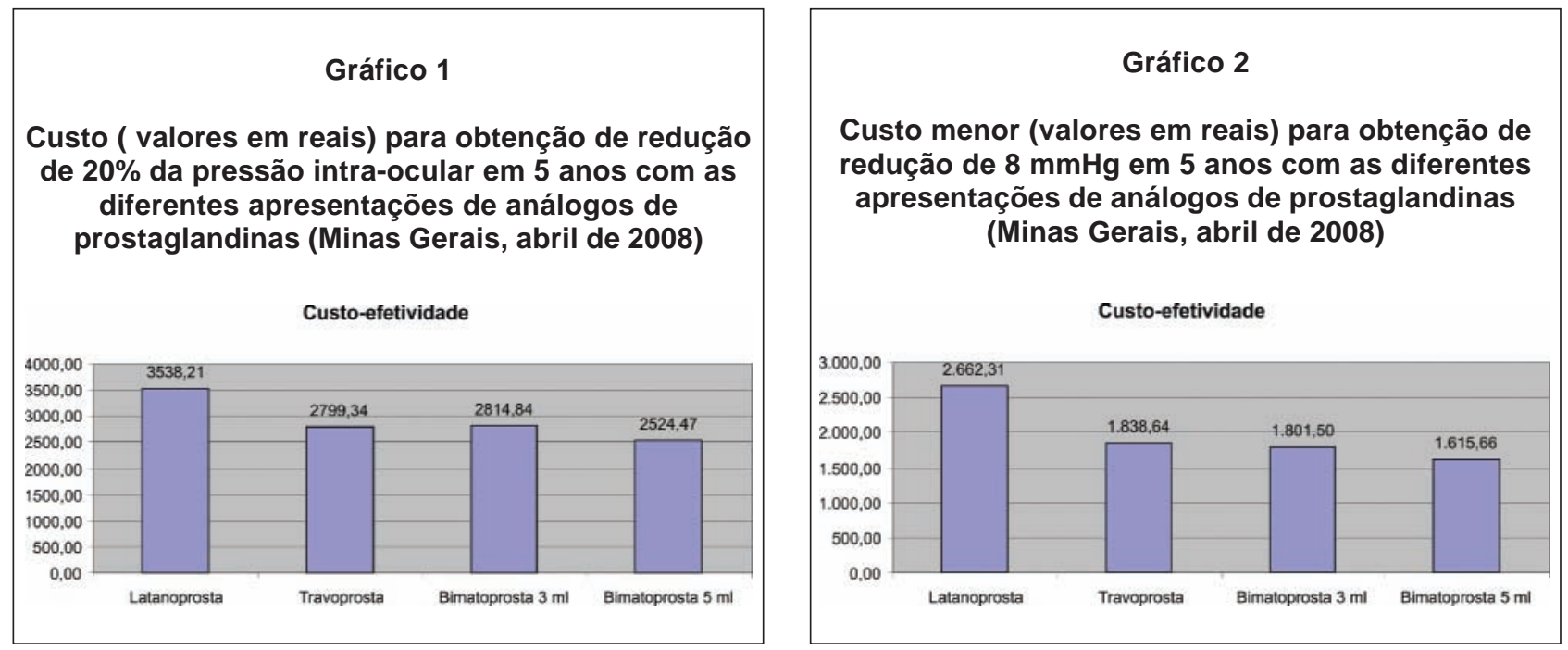

Tabela 4

Custo-efetividade dos análogos de prostaglandinas avaliando-se a porcentagem média de redução pressórica (Minas Gerais, abril de 2008)

\begin{tabular}{lccc|ccc}
\hline & \multicolumn{3}{c|}{ Redução de 1\% da PIO } & \multicolumn{3}{c}{ Redução de 20\% da PIO } \\
\cline { 2 - 7 } Medicamentos & $\begin{array}{c}\text { Custo } \\
\text { mensal }\end{array}$ & $\begin{array}{c}\text { Custo } \\
\text { anual }\end{array}$ & $\begin{array}{c}\text { Custo } \\
\text { em 5 anos }\end{array}$ & $\begin{array}{c}\text { Custo } \\
\text { mensal }\end{array}$ & $\begin{array}{c}\text { Custo } \\
\text { anual }\end{array}$ & $\begin{array}{c}\text { Custo } \\
\text { em 5 anos }\end{array}$ \\
\hline Latanoprosta & 2,22 & 26,62 & 133,12 & 44,37 & 532,46 & $2.662,31$ \\
Travoprosta & 1,53 & 18,39 & 91,93 & 30,64 & 367,73 & $1.838,64$ \\
Bimatoprosta 3 ml & 1,50 & 18,01 & 90,07 & 30,02 & 360,30 & $1.801,50$ \\
Bimatoprosta 5 ml & 1,35 & 16,16 & 80,78 & 26,93 & 323,13 & $1.615,66$ \\
\hline
\end{tabular}

Cálculo utilizando o preço máximo ao consumidor (abril 2008) e média de redução da pressão intra-ocular (PIO) segundo Denis et al. ${ }^{(10)}$;

Custo = Valores em reais;

$\mathrm{PIO}=$ Pressão intra-ocular

Tabela 5

Custo-efetividade dos análogos de prostaglandinas avaliando-se os limites de redução da pressão intra-ocular (PIO) propostos por Frenkel et al. (11) (Minas Gerais, abril de 2008)

\begin{tabular}{|c|c|c|c|c|c|c|}
\hline \multirow[b]{2}{*}{ Medicamentos } & \multicolumn{3}{|c|}{ Redução de 1 mmHg da PIO } & \multicolumn{3}{|c|}{ Redução de 8 mmHg da PIO } \\
\hline & $\begin{array}{l}\text { Custo } \\
\text { mensal }\end{array}$ & $\begin{array}{l}\text { Custo } \\
\text { anual }\end{array}$ & $\begin{array}{c}\text { Custo } \\
\text { em } 5 \text { anos }\end{array}$ & $\begin{array}{c}\text { Custo } \\
\text { mensal }\end{array}$ & $\begin{array}{c}\text { Custo } \\
\text { anual }\end{array}$ & $\begin{array}{c}\text { Custo } \\
\text { em } 5 \text { anos }\end{array}$ \\
\hline Latanoprosta & $7,37 \mathrm{a}^{* *}$ & 88,46 a & $442,28 \mathrm{a}$ & 58,97 a & $707,64 \mathrm{a}$ & $3.538,21 \mathrm{a}$ \\
\hline (redução de 6 a 8 mmHg)* & 9,83 & 117,94 & 589,70 & 78,64 & 943,52 & $4.717,60$ \\
\hline Travoprosta & $5,83 \mathrm{a}$ & $69,98 \mathrm{a}$ & $349,92 \mathrm{a}$ & $46,66 \mathrm{a}$ & 559,87 a & $2.799,34 \mathrm{a}$ \\
\hline (redução de 7 a 8 mmHg)* & 6,67 & 79,98 & 399,91 & 53,36 & 639,84 & $3.199,28$ \\
\hline Bimatoprosta de $3 \mathrm{ml}$ & 5,86 a & $70,37 \mathrm{a}$ & $351,85 \mathrm{a}$ & $46,91 \mathrm{a}$ & 562,97 a & $2.814,84 \mathrm{a}$ \\
\hline (redução de 7 a $8 \mathrm{mmHg}$ )* & 6,70 & 80,42 & 402,12 & 53,60 & 643,36 & $3.216,96$ \\
\hline Bimatoprosta de $5 \mathrm{ml}$ & $5,26 \mathrm{a}$ & $63,11 \mathrm{a}$ & $315,56 \mathrm{a}$ & $42,07 \mathrm{a}$ & 504,89 a & $2.524,47 \mathrm{a}$ \\
\hline (redução de 7 a $8 \mathrm{mmHg}$ )* & 6,01 & 72,13 & 360,64 & 48,08 & 577,04 & $2.885,12$ \\
\hline
\end{tabular}

* Valores obtidos de Frenkel et al. (11);

** Variação do custo obtida através da divisão do custo do colírio pela capacidade de redução pressórica;

Custo $=$ Valores em reais 
va seria ainda a bimatoprosta de $5 \mathrm{ml}$. No entanto, no segundo lugar estaria a travoprosta, seguida pela bimatoprosta de $3 \mathrm{ml}$ e por último pela latanoprosta.

\section{DiscussÃo}

O custo pode influenciar decisivamente na tomada de decisões para o tratamento do glaucoma. Nos EUA, os custos do tratamento do glaucoma têm uma estimativa de 2,5 bilhões de dólares por ano, com 1,9 bilhões de custos diretos e 0,6 bilhões de custos indiretos ${ }^{(13)}$. Os custos diretos do glaucoma representam um ônus significativo no orçamento global da saúde ${ }^{(14)}$. Eles incluem, segundo Doshi e Singh ${ }^{(3)}$, tratamento medicamentoso, cirúrgico, consultas médicas, hospitalizações e exames complementares. Em 2006, o Center for Disease Control and Prevention estimou em 1,8 bilhões de dólares os custos diretos com glaucoma em pacientes acima de 40 anos ${ }^{(15)}$. Os custos das medicações antiglaucomatosas têm estimativas que compreendem de 38 a $52 \%$ do total de custos diretos ${ }^{(16)}$. Estes custos sofrem influência direta da duração de cada frasco (número de gotas por fras$\mathrm{co})^{(17)}$. Na presente investigação, o número médio de gotas de cada frasco foi obtido de estudos prévios brasileiros ${ }^{(6-9)}$. Em análise recente nos EUA, Rylander e Vold ${ }^{(1)}$ estimaram o custo anual para o paciente em uso crônico de colírios. Os valores com o uso de uma única medicação variaram de 150,81 até 873,98 dólares americanos por ano. Isto sem levar em conta a necessidade de se associar dois ou mais medicamentos em quase metade dos pacientes ${ }^{(12)}$.

O envelhecimento da população mundial e o conseqüente aumento da prevalência do glaucoma requerem uma alocação custo-efetiva de recursos no tratamento e no controle desta doença ${ }^{(3)}$.Além das tradicionais avaliações de segurança e eficácia dos medicamentos, a análise de custo-efetividade deveria ser considerada para auxiliar na tomada de decisões individuais e coletivas ${ }^{(18)}$. Na avaliação da relação entre o custo e a efetividade verificar-se-ia, do ponto de vista prático, qual a droga mais adequada para o sistema de saúde como um todo, devendo, no entanto, o tratamento ser individualizado para cada paciente.

No presente estudo, em ambas as análises (redução da pressão em valores relativos ou absolutos) a medicação mais custo-efetiva foi a bimatoprosta em frasco de $5 \mathrm{ml}$. O volume do frasco maior faz com que o preço por gota caia, tornando a medicação mais barata. Com a finalidade de proporcionar uma melhora da relação custo-efetividade, todas as indústrias farmacêuticas deveriam ser estimuladas a fazer apresentações com um volume maior.

Em uma comparação direta entre as três apresentações mais comuns e com volumes similares (latanoprosta de 2,5 ml; travoprosta de 2,5 $\mathrm{ml} \mathrm{e}$ bimatoprosta de $3,0 \mathrm{ml}$ ), observa-se que a medicação mais custo-efetiva varia de acordo com o critério de redução pressórica escolhido.

Se utilizarmos a redução pressórica em termos percentuais, baseando-se na meta-análise feita por Denis et al. ${ }^{(10)}$, a droga mais custo-efetiva seria a bimatoprosta, seguida da travoprosta e da latanoprosta. A diferença entre as duas primeiras foi muito pequena ( $1 \%$ de redução da $\mathrm{PIO} / \mathrm{mês}: 1,53$ reais para a travoprosta e 1,50 reais para a bimatoprosta). Já a latanoprosta tem a pior relação custo-efetividade, apresentando uma diferença significativa em relação as demais. Esta diferença pode chegar a mais de 800 reais em acréscimo do custo em 5 anos, para uma redução de $20 \%$ da PIO inicial.

Se a análise é feita avaliando-se a redução pressórica em unidades ( $\mathrm{mmHg}$ ), a medicação mais custo-efetiva passa a ser a travoprosta, seguida de perto pela bimatoprosta de $3 \mathrm{ml}$ e depois pela latanoprosta. Mais uma vez, a diferença entre as duas primeiras é muito pequena. O custo máximo para se reduzir $1 \mathrm{mmHg}$ da PIO por mês seria de 6,67 reais para a travoprosta e de 6,70 reais para a bimatoprosta de $3 \mathrm{ml}$. O mesmo custo para a latanoprosta seria muito maior ( 9,83 reais). Da mesma forma, a diferença em um horizonte de 5 anos se torna bem mais evidente. A utilização da latanoprosta poderia gerar um custo adicional de quase 800 reais em relação as outras duas prostaglandinas, para uma redução de 8 mmHg em 5 anos.

Nos Estados Unidos, estudos semelhantes encontraram que a medicação mais custo-efetiva seria a bimatoprosta, seguida da travoprosta e da latanoprosta ${ }^{(10-18)}$.A extrapolação e comparação dos resultados ficam difíceis de serem feitas, pois as realidades são diferentes (custo principalmente).

Pelo presente estudo, a relação custo-efetividade sofre grande influência do volume do frasco. Quanto maior o volume deste, mais custo-efetiva é a medicação. Ao se analisar as medicações com volumes similares, observa-se que a travoprosta e a bimatoprosta tem uma relação custo-efetividade bem semelhante. Por outro lado, a latanoprosta foi, em todas as análises, a medicação mais cara.

O estudo da relação custo-efetividade possui limitações, pois avalia somente um aspecto do complexo sistema para a escolha da melhor medicação do ponto de vista individual. Outros fatores devem ser levados em consideração, tais como: efeitos colaterais dos medicamentos, taxa de fidelidade, taxa de descontinuidade, taxa de não respondedores, etc. Um estudo que poderia ser importante ao avaliar, pelo menos parcialmente, a qualidade de vida proporcionada pelo tratamento em relação ao custo e à efetividade seria da relação custoutilidade das prostaglandinas. 
Os estudos de custo-efetividade são de grande valia para o sistema público de saúde, pois podem auxiliar na tomada de decisões sobre qual medicação padronizar como primeira escolha em um centro de referência. Faz-se mister salientar que, isto não quer dizer que a medicação mais custo-efetiva será sempre a melhor. A escolha deve ser sempre pautada em uma análise pormenorizada e individualizada de cada paciente. No caso de não haver impedimento ou contra-indicação, dar-seia preferência àquela mais custo-efetiva.

\section{ConClusão}

A medicação mais custo-efetiva para o tratamento do glaucoma e da hipertensão ocular no estado de Minas Gerais, no Brasil, foi a bimatoprosta na apresentação de $5 \mathrm{ml}$. A latanoprosta foi a medicação com pior relação custo-efetividade. A bimatoprosta na apresentação de 3 $\mathrm{ml}$ e a travoprosta apresentaram resultados similares, ficando em posição intermediária entre as duas anteriores.

\section{Abstract}

Purpose: Assess cost-effectiveness of glaucoma and/ or ocular hypertension medical therapy using prostaglandin analogues in the state of Minas Gerais, Brazil. Methods: This cross-sectional study evaluated the cost (average wholesale price) of different prostaglandin analogues (bimatoprost, latanoprost, travoprost) in relation to its effectiveness in reducing intra-ocular pressure. Monthly, annually and 5-year cost to achieve $1 \%$ and $20 \%$ of IOP reduction was calculated. Monthly, annually and 5-year cost to reduce $1 \mathrm{mmHg}$ and $8 \mathrm{mmHg}$ from baseline IOP was also calculated. Results: Monthly cost to achieve 1\% and $20 \%$ of IOP reduction was, respectively, $R \$ 1.35$ and $R \$ 27.00$ for bimatoprost $5 \mathrm{ml}, R \$ 1.50$ and $R \$$ 30.00 for bimatoprost $3 \mathrm{ml}, R \$ 1.53$ and $R \$ 30.60$ for travoprost and $R \$ 2.22$ and $R \$ 44.40$ for latanoprost. Monthly maximum cost to reduce baseline IOP 1 $\mathrm{mmHg}$ and $8 \mathrm{mmHg}$ was, respectively, $R \$ 6.01$ and $R \$ 48.08$ for bimatoprost $5 \mathrm{ml}, R \$ 6.67$ and $R \$ 53.36$ for travoprost, $R \$ 6.70$ and $R \$ 53.60$ for bimatoprost $3 \mathrm{ml}$ and $R \$ 9.83$ and $R \$ 78.64$ for latanoprost. Conclusion: Cost-effectiveness was better for bimatoprost $5 \mathrm{ml}$. The medication, which had the worst cost-effectiveness relationship, was latanoprost. Travoprost and bimatoprost $3 \mathrm{ml}$ showed similar and intermediate results.

Keywords: Glaucoma/drug therapy; Intraocular pressure/drug therapy; Prostaglandins, synthetic/ economics; Pharmacoeconomics; Drug costs; Costeffectiveness evaluation; Costs and cost analysis; Brazil

\section{REFERÊNCIAS}

1. Rylander NR, Vold SD. Cost analysis of glaucoma medications. Am J Ophthalmol. 2008;145(1):106-13.

2. Vianna CM, Caetano R. Avaliação tecnológica em saúde: introdução a conceitos básicos. Rio de Janeiro: UERJ; 2001.

3. Doshi A, Singh K. Cost-effective evaluation of the glaucoma suspect. Curr Opin Ophthalmol. 2007;18(2):97-103.

4. Finkler SA. The distinction between cost and charges. Ann Intern Med. 1982;96(1):102-9.

5. Gold MR, Russel LB, Weinstein MC. Cost-effectiveness in health and medicine. New York: Oxford University; 1996.

6. Stillitano IG, Tenório A, Cardoso G, Ribeiro MP, Figueiroa JN. Custo do tratamento de drogas antiglaucomatosas: iatanoprost, travoprost, bimatoprost, unoprostona isopropílica. Arq Bras Oftalm. 2003; 66(6):859-64.

7. Stillitano IG, Lima MG, Ribeiro MP, Cabral J, Brandt CT. Impacto econômico do custo de colírios no tratamento do glaucoma. Arq Bras Oftalmol. 2005; 68(1):79-84.

8. Galvão-Neto P, Rocha Júnior FS, Ribeiro BB, Barreto B, Silva FA, Figueiredo $C R$ et al. Volume da gota dos análogos das prostaglandinas. Rev Bras Oftalmol. 2004; 63(9/10): 457-62.

9. Galvão Neto P, Rocha Júnior FS, Ribeiro BB, Figueiredo CR, Galvão RP. Custo mensal de hipotensores oculares em Belo Horizonte - MG. Rev Bras Oftalmol. 2003;62(9):653-59.

10. Denis P, Lafuma A, Khoshnood B, Mimaud V, Berdeaux G. A meta-analysis of topical prostaglandin analogues intra-ocular pressure lowering in glaucoma therapy. Curr Med Res Opin. 2007;23(3):601-8.

11. Frenkel RE, Frenkel M, Toler A. Pharmacoeconomic analysis of prostaglandin and prostamide therapy for patients with glaucoma or ocular hypertension. BMC Ophthalmol. 2007;7:16.

12. Kass MA, Heuer DK, Higginbotham EJ, Johnson CA, Keltner JL, Miller JP, et al. The Ocular Hypertension Treatment Study: a randomized trial determines that topical ocular hypotensive medication delays or prevents the onset of primary openangle glaucoma. Arch Ophthalmol. 2002;120(6):701-13.

13. Glick H, Brainsky A, McDonald RC, Javitt JC. The cost of glaucoma in the United States in 1988. Chibret Int J Ophthalmol. 1994; 10: 6-12.

14. Lee PP, Levin LA, Walt JG, Chiang T, Katz LM, Dolgitser M, et al. Cost of patients with primary open-angle glaucoma: a retrospective study of commercial insurance claims data. Ophthalmology. 2007;114(7):1241-7.

15. Rein DB, Zhang P, Wirth KE, Lee PP, Hoerger TJ, McCall N, et al. The economic burden of major adult visual disorders in the United States. Arch Ophthalmol. 2006;124(12):1754-60.

16. Lee PP, Walt JG, Doyle JJ, Kotak SV, Evans SJ, Budenz DL, et al. A multicenter, retrospective pilot study of resource use and costs associated with severity of disease in glaucoma. Arch Ophthalmol. 2006;124(1):12-9.

17. Vaidergorn PG, Susanna Júnior R, Borges AS, Giampani Júnior J. Tempo de terapêutica propiciado por frascos de colírios hipotensores oculares. Rev Bras Oftamolol. 2002;61(4):278-82.

18. Noecker RJ, Walt JG. Cost-effectiveness of monotherapy treatment of glaucoma and ocular hypertension with the lipid class of medications. Am J Ophthalmol. 2006;141(1 Suppl):S15-21.
ENDEREÇO PARA CORRESPONDÊNCIA:
Dr. Ricardo Augusto Paletta Guedes
Av. Rio Branco 2337 - grupo 801/807/808
Centro - Juiz de Fora - MG
Telefax: (32) 32131927
E-mail: palettaguedes@yahoo.com 